\title{
Measurement Matrix Construction Algorithm for Compressed Sensing based on QC-LDPC Matrix
}

\author{
NIE Yang ${ }^{1,2}$ and JING Li-li ${ }^{1}$ \\ ${ }^{1}$ Department of Physics, Jining Normal University, Inner Mongolia, China \\ ${ }^{2}$ Digital Engineering Center, Communication University of China, Beijing, China \\ nieyangwork@163.com
}

\begin{abstract}
The measurement matrix of compressed sensing has a significant impact for sampling and reconstruction algorithm of the original signal. At present, the majority of the measurement matrix is randomly constructed, and it is difficulty for hardware implementation in the practical applications. In this paper, we use the sparse characteristic of parity- check matrix of LDPC codes, construct measurement matrix based on QC-LDPC (Quasi-cyclic low-density parity-check) matrix, which is a structural and sparse deterministic measurement matrix. The simulation results show that, the measurement matrix is proposed in this paper not only can obtain a better reconstructed image quality, but also it can reduce the complexity of hardware implementation for quasi-cyclic.
\end{abstract}

Keywords: compressed sensing; measurement matrix; QC-LDPC; image compression

\section{Introduction}

According to sampling theory requirement, if the frequency of sampling signal is at least twice the signal bandwidth, it can be completely reconstructed. The entire process of signal sampling is shown in Figure 1. But, in the digital image and video processing system, the sampling frequency of the signal is too high, get a lot of sample values. Before processing and sending signal, it must be compressed, which will waste a lot of storage space and sampling devices. With increasing to the information demand, the bandwidth of the signal is more and more wide, sampling rate and processing speed more and more is also high in the traditional signal processing framework. Therefore, big data signal data transmission and storage to be a hard job.

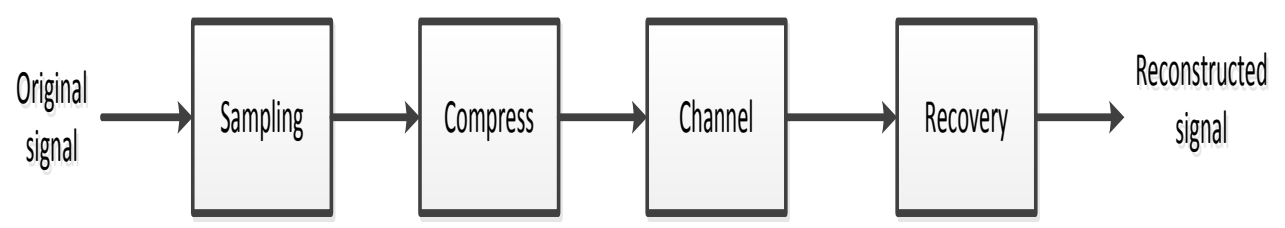

Figure 1. Conventional Signal Sampling Process

In recent years, there is an innovative signal processing theory: CS (Compressed Sensing, CS $)^{[1-2]}$, which is a new signal processing mode, and the entire process is shown in Figure 2. Compressed sensing theory is different to sampling theory. Theory study ${ }^{[3]}$ show that if the signal is sparse in a transform domain, and transform the highdimensional data onto a low

dimensional space with measurement matrix, which is not related to transform base. Then this small amount of projection can be reconstructed the original signal with high probability by solving an optimization problems. 


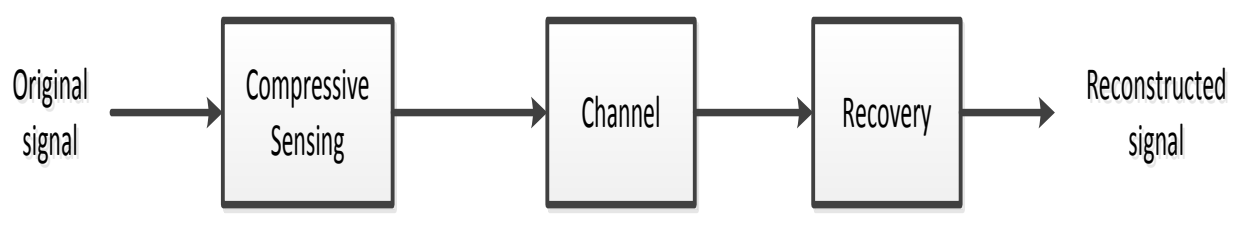

Figure 2. Signal Sampling Process based on CS

Currently, the core issue of CS study includes two aspects: the measurement of signal; reconstruction algorithm of signal. The measurement signal is determined by measurement matrix. Theoretically, the measurement matrix must be meet the RIP (Restricted Isometry Property, RIP) ${ }^{[4]}$ to ensure the reconstruction of the original signal from a small number of measurement signal.

Gauss matrix and the part of the Fourier matrix has been proved to meet RIP feature in high probability .However, due to Gaussian matrix is random matrix, therefore it has a lot of storage space, and is high computational complexity for unstructured. The computational complexity of the Part of Fourier matrix has improved, but the orthogonal has weakened compared with the Gaussian matrix .The performance of sparse random matrix based on extended code has the same as Gaussian random matrix, but due to the random structure, the orthogonality of the matrix can't be controlled. The measurement matrix, especially the random measurement matrix, although there are some advantages on performance, but the hardware implementation is difficult. Therefore, the structured measurement matrix is becoming a hot spot of research. LDPC Code (Low Density Parity Check Code) is a kind of linear block codes, has the excellent properties of approaching Shannon limit. The parity check matrix is sparse binary matrix, compared with the existing measurement matrix, which has three advantages as the measurement matrix: the orthogonality of parity check matrix is strong; The related algorithm can control the orthogonality of the matrix; The structure of parity check matrix is determined, and belong to the sparse matrix.

In this paper, we propose a construction method of deterministic measurement matrix for compressed sensing by the sparse and quasi-cycle of QC-LDPC parity check matrix. Section 2 structures measurement matrix based on QC-LDPC. Section 3 provides numerical simulation and verifies the validity of the new measurement matrix. Section 4 concludes the conclusion.

\section{The Construction of Measurement Matrix}

\subsection{Restricted Isometry Property}

In the compressed sensing, $x$ is an $M \times N$ column vector in $\mathrm{R}^{N} . x$ is not generally sparse signals, but it is sparse in the transform base $\Psi$, so it can be expressed as

$x=\Psi s$

Where $s$ is the $N \times 1$ column vector, if $s$ has $K$ nonzero term, then it can be called $K-$ sparse.

Measurement matrix is a matrix $\Phi$. $y$ is a collection of measurement vector, and $y$ can be written as

$y=\Phi \Psi s=\Theta s$ 
$\Theta=\Phi \Psi$ is an $M \times N$ matrix, It is called sensor matrix. Candes and Tao ${ }^{[5]}$ presented and proved that if the sensor matrix $\Theta$ satisfies RIP, $x$ can be reconstructed by $y$ with a high probability. RIP is defined as follows. For each integer $K=1,2, \ldots \ldots$, define the isometry constant $\delta_{K}$ of $\Theta$ as the smallest number such that

$$
\left(1-\delta_{K}\right)\|s\|_{l_{2}}^{2} \leq\|\Theta s\|_{l_{2}}^{2} \leq\left(1+\delta_{K}\right)\|s\|_{l_{2}}^{2}
$$

Donoho presented the concept of compressed sensing at the same time, and provided three characteristics of measurement matrix: the column vector of measurement matrix satisfies linear independence; the column vector of measurement matrix can reflect similar with independence of random noise; the solution of sparse is to meet the minimum $l_{0}$ norm. In addition, TROPPJA studies have shown that, if they can meet the change between the base and the measurement matrix sparse uncorrelated, and then the probability of the measurement matrix will be a great meet RIP nature. If it is non-linear correlation between transform base and measurement matrix, then the measurement matrix will be meet RIP nature by the high probability.

\subsection{The Parity Check Matrix of LDPC}

LDPC codes $^{[6]}$ are one kind of linear block codes, which are defined in the null space of sparse binary parity-check matrix .To evaluate their structure and performance, paritycheck matrix is often represented and analyzed by Tanner graph. For example, Figure.3b shows a Tanner graph associated with parity-check matrix H in Figure.3a.In this Tanner graph, the upper five circles are often called variable nodes, sequentially corresponding to the five columns of matrix $\mathrm{H}$. And the lower four squares are called check nodes, corresponding to the four rows of $\mathrm{H}$. The edges between two classes of nodes are rendered by the nonzero entries in $\mathrm{H}$. As shown in Figure.3c, the gray circle denotes the root of a tree. In this tree, there are three cycles passing through the root variable node, and they respectively include 4, 6 and 8 edges.

$$
H=\left[\begin{array}{llllll}
1 & 0 & 1 & 0 & 1 & 0 \\
1 & 0 & 0 & 1 & 0 & 1 \\
0 & 1 & 1 & 0 & 1 & 1 \\
0 & 1 & 0 & 1 & 0 & 0
\end{array}\right]
$$

(a)

(b)

(c)

Figure 3. Parity-Check Matrix of LDPC and Tanner Graph

Theoretically, the trees with bigger cycles are preferred because it means that the root variable node keeps higher independency with other variable nodes. Specially, for LDPC codes, the cycle with 4 edges, e.g. the cycle with dotted lines in Figure.3c, is usually avoided for iterative decoding. In other words, the columns of matrix mutually hold lower correlation in structure, which are also the intrinsic requirements of RIP.

\subsection{The Parity Check Matrix of QC-LDPC}

The parity check matrix of QC-LDPC ${ }^{[7]}$ is based on cyclic shift matrices, which are composed of identity matrix. An $m s \times n s$ parity check matrix of QC-LDPC can be written as 


$$
H=\left[\begin{array}{cccc}
I\left(S_{11}\right) & I\left(S_{12}\right) & \cdots & I\left(S_{1 n}\right) \\
I\left(S_{21}\right) & I\left(S_{22}\right) & \cdots & I\left(S_{2 n}\right) \\
\vdots & \vdots & \ddots & \vdots \\
I\left(S_{m 1}\right) & I\left(S_{m 2}\right) & \cdots & I\left(S_{m n}\right)
\end{array}\right]
$$

Where $I\left(S_{i j}\right)(1 \leq i \leq m, 1 \leq j \leq n)$ is the $s \times s$ rotate right matrix, which is called cyclic permutation sub matrix. The $s \times s$ identity matrix is shift right by $S_{i j}$, where $S_{i j} \in\{0,1,2, \cdots, p-1, \infty\}$, if $S_{i j}=0$, It represents $I\left(S_{i j}\right)$ is an identity matrix; if $S_{i j}=\infty$,It represents $I\left(S_{i j}\right)$ is an zero matrix.

If the cyclic permutation sub-matrix $I\left(S_{i j}\right)$ of the check matrix $\mathrm{H}$ is expressed by cyclic shift number $S_{i j}$, then parameter shift matrix $S$ is obtained, it can be written as

$$
S=\left[\begin{array}{cccc}
S_{11} & S_{12} & \cdots & S_{1 n} \\
S_{21} & S_{22} & \cdots & S_{2 n} \\
\vdots & \vdots & \ddots & \vdots \\
S_{m 1} & S_{m 2} & \cdots & S_{m n}
\end{array}\right]
$$

If non-infinite is replaced by ' 1 ' in the parameter shift matrix $S$, and infinite is replaced by ' 0 ', the $m \times n$ basis matrix $U$ is obtained, where $U_{i j} \in\{0,1\}, \quad(1 \leq i \leq m, 1 \leq j \leq n)$, it can be written as

$$
U=\left[\begin{array}{cccc}
U_{11} & U_{12} & \cdots & U_{1 n} \\
U_{21} & U_{22} & \cdots & U_{2 n} \\
\vdots & \vdots & \ddots & \vdots \\
U_{m 1} & U_{m 2} & \cdots & U_{m n}
\end{array}\right]
$$

Through the above analysis shows that, when the base matrix $U$ and parameter shift matrix $S$ are determined, the parity check matrix $H$ of QC-LDPC can also be determined.

\subsection{Design of QC-LDPC Matrix based on PEG Algorithm}

PEG (progress edge growth) ${ }^{[8]}$ algorithm is adopted to structure Tanner graph by the edge growth. When the edge is added, it makes the ring of the node is the largest long, and makes the number of the short loop as little as possible and the whole ring as large as possible in Tanner graph. Therefore, using PEG algorithm is constructed LDPC code is called "currently know the best method to construct LDPC code". But because of result of random structures, the check matrix is lack of structural, and need a lot of storage unit to store check matrix.

The check matrix of QC-LDPC has a less storage unit and easy to realize based on PEG algorithm. In this algorithm, four initial variables, the number of check nodes $(m)$, the number of variable nodes $(n)$, the degree of variable nodes $(d)$ and cyclic permutation submatrix $(p)$ are entered. The algorithm is summarized as follows

$$
\begin{gathered}
\text { for } j=0 \text { to } n-1 \text { do } \\
\text { for } k=0 \text { to } d_{v_{j}} \text { do } \\
\quad \text { if } k=0
\end{gathered}
$$

Initial the edges for any variable nodes by selecting the minimum degree of check nodes, then establish the edge as $1^{\text {st }}$ edge. 
else

Insert other edges to expand tree diagram from $1^{s t}$ edge under the current graph up to $l$-depth as the following rule (Figure. 4$) . \overline{N_{v_{j}}^{l}} \neq 0$

If $\overline{N_{v_{j}}^{l}} \neq 0$, but $\overline{N_{v_{j}}^{l}} \neq \phi$, select the check node that has the largest distance from $v_{j}, N_{v_{j}}^{l}$ represents the check nodes which can be reached from $v_{j}$ at a $l$-depth. The complementary of $N_{v_{j}}^{l}$ is $\overline{N_{v_{j}}^{l}}$. If cardinality of $N_{v_{j}}^{l}$ stops increasing but is smaller than $m$, select the check node unreachable from $v_{j}$ to establish the next edge. end

Create cyclic permutation sub-matrix based on the edges in the previous step. Then we add cyclic permutation sub-matrix to the check matrix instead of node end by node. If the element is zero, we place the null matrix.

end

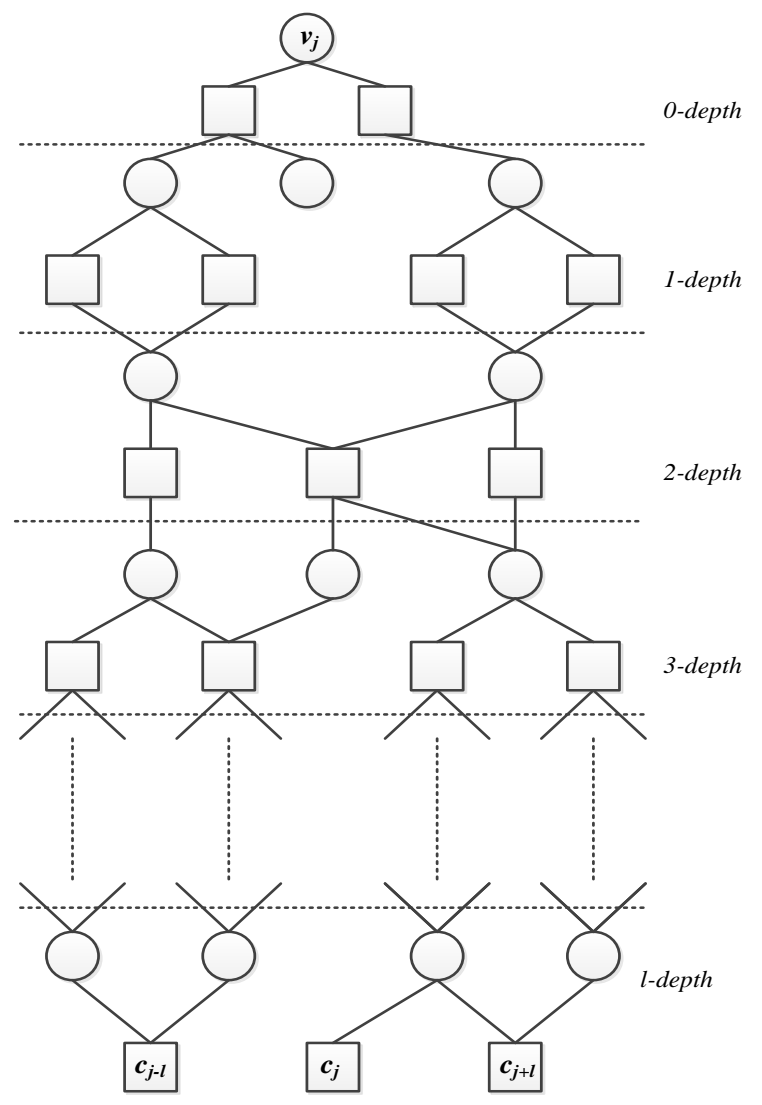

Figure 4.Tree Diagram Expanding from Variable Node

\section{The Simulation Results and Discussions}

Theory analysis ${ }^{[9-11]}$ shows that: if a binary parity check matrix has a good performance in the channel coding and decoding, then the matrix also has a good performance as a measurement matrix for CS. In this paper, we will use one-dimensional and twodimensional signals for the test object, and verify the performance of check matrix in the CS. 


\subsection{The Simulation and Analysis of One-Dimensional Signal}

If $x$ is one dimensional signal, which is made up of four different frequencies sine signals.

$x=\cos (2 \pi \times t s / 128)+\sin (2 \pi \times t s / 256)+\sin (2 \pi \times t s / 64)+\cos (2 \pi \times t s / 32)$, The time domain waveform of one-dimensional signal $x$ is shown in Figure. 5 and it is non-parse. But it has limited non-zero value in frequency domain, which is shown in Figure 6.

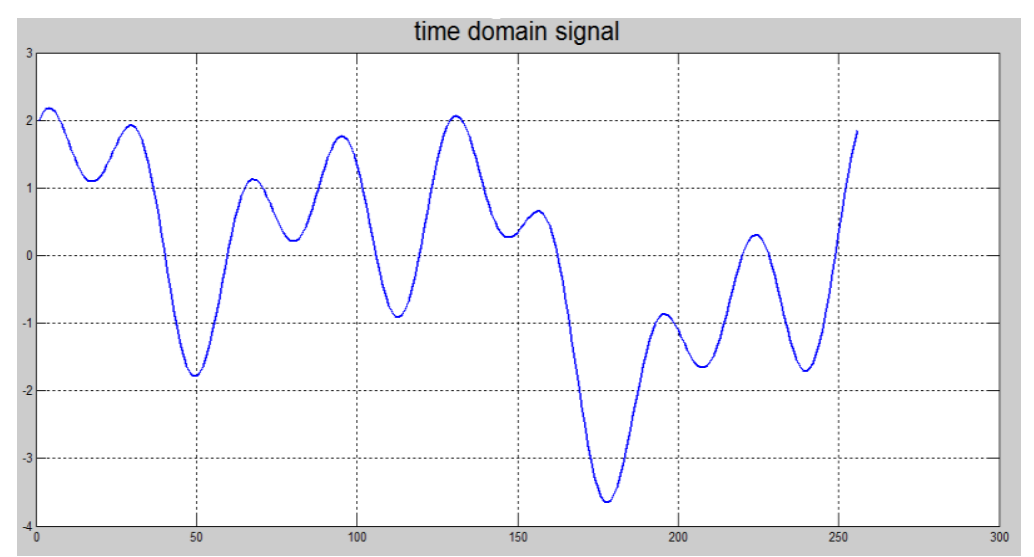

Figure 5.The Time Domain Waveform of One-Dimensional Signal

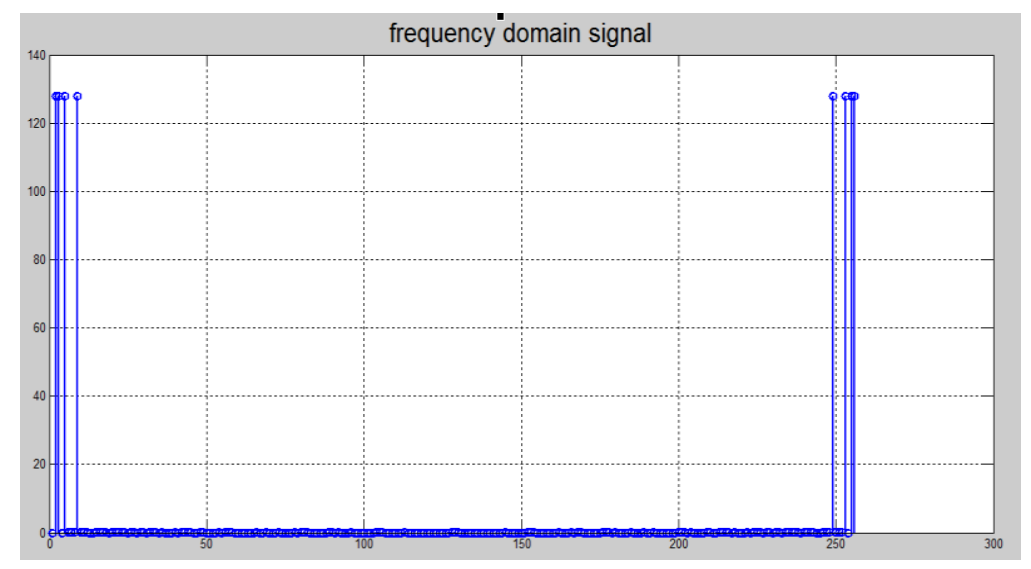

Figure 6. The Frequency Domain Waveform of One-Dimensional Signal

If the signal $x$ is selected as the test signal, compression ratio $r=M / N=0.5$, and reconstruction algorithm is used the $\mathrm{OMP}^{[12-14]}$ algorithm, and choose different measurement matrix such as Gauss matrix, Bernoulli matrix, part of Hadamard matrix, sparse random matrix , LDPC-PEG matrix, QC-LDPC matrix, and the simulation result is shown in Figure 7. 


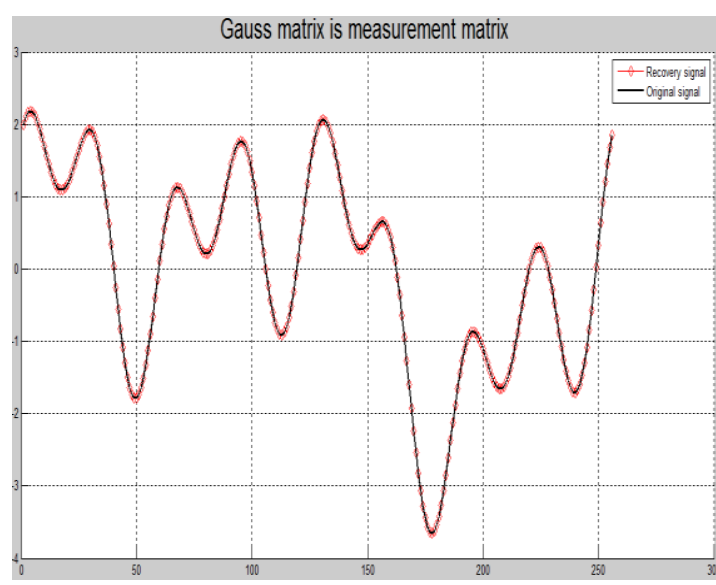

(a)

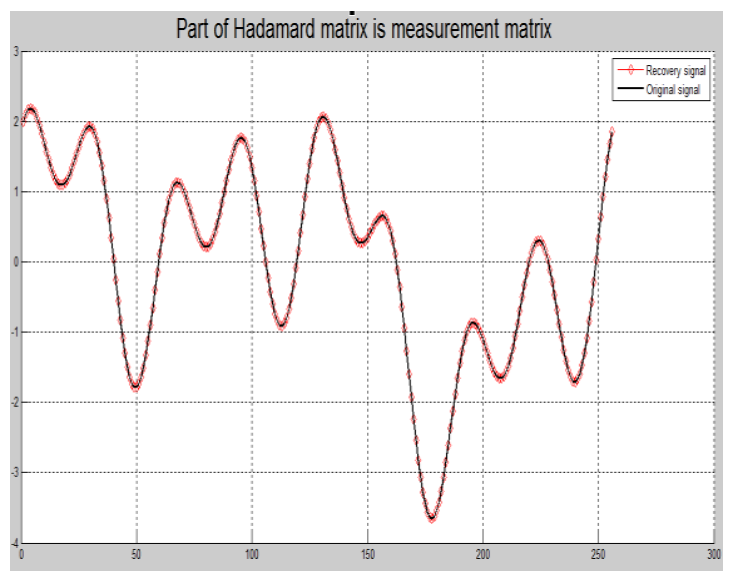

(c)

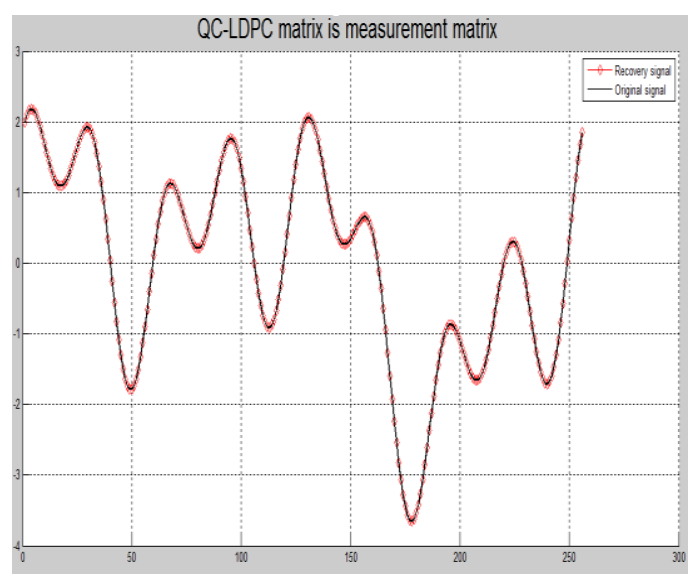

(e)

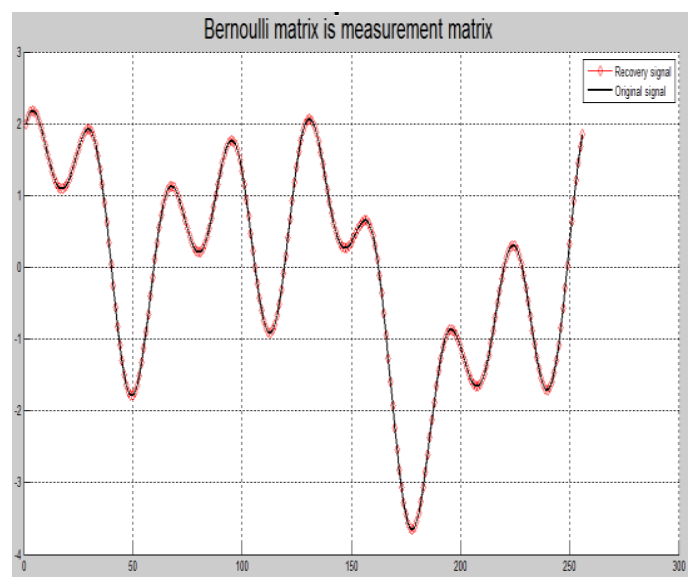

(b)

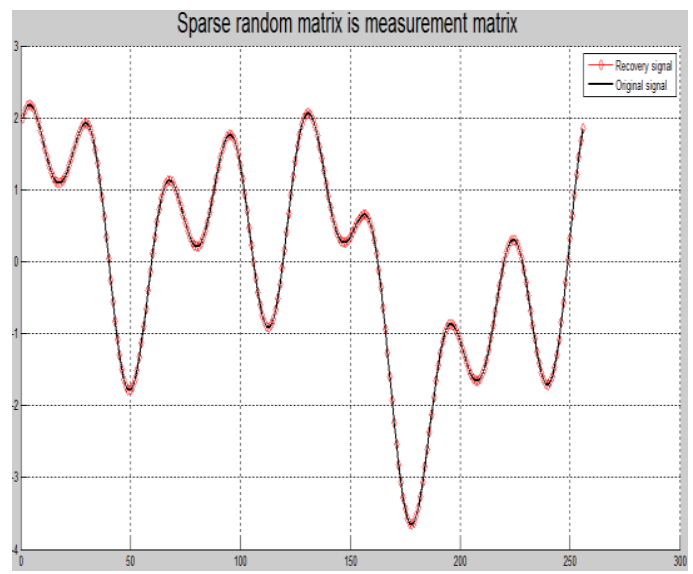

(d)

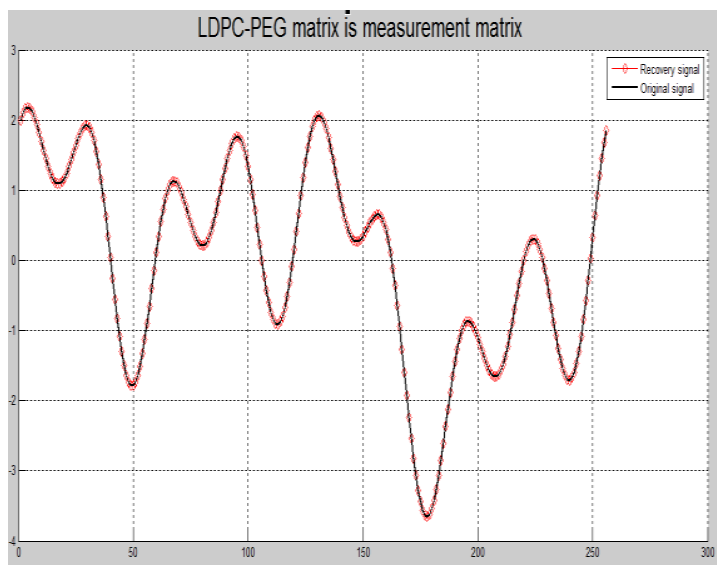

(f)

Figure 7. One-Dimensional Signal Reconstruction by Measurement Matrixes

The simulation results can be seen from the Figure 7, and the reconstruction effect of signal $\mathrm{x}$ is very ideal. The error comparison are shown in Table 1 , the orders of magnitude are at $10^{-15}$. Gauss matrix, Bernoulli matrix, part of Hadamard matrix, sparse random matrix are used as a measurement matrix, the effect is essentially the same. But these matrix structure are randomly constructed and hardware implementation are very difficult [15][16]. The QC-LDPC matrix proposed in this paper is the structure determination and better performance, so it is ideal for the measurement matrix. 
Table 1. The Error Comparison of the Reconstruction Measurement Matrix

\begin{tabular}{ccc}
\hline Measurement matrix & Error & Compression ratio \\
\hline Gauss matrix & $1.0506 \mathrm{e}^{-15}$ & 0.5 \\
\hline Bernoulli matrix & $1.1251 \mathrm{e}^{-15}$ & 0.5 \\
\hline part of Hadamard matrix & $1.0415 \mathrm{e}^{-15}$ & 0.5 \\
\hline Sparse random matrix & $1.0288 \mathrm{e}^{-15}$ & 0.5 \\
\hline LDPC-PEG & $2.0506 \mathrm{e}^{-15}$ & 0.5 \\
\hline QC-LDPCmatrix & $1.2506 \mathrm{e}^{-15}$ & 0.5 \\
\hline
\end{tabular}

\subsection{The Simulation and Analysis of Two-Dimensional Signal}

Lena Figure was used as test object, and its size is $256 \times 256$.To facilitate processing, Lena Figure can be divided into $16 \times 16$ pieces for processing. The original image is transformed by DCT ${ }^{[17]}{ }^{[18]}$, and retain $K$ maximum coefficient of wavelet, then the coefficient of each column is measured and reconstructed separately. Compression ratio $r=M / N=0.5$, and reconstruction algorithm is used the OMP algorithm, and choose different measurement matrix such as Gauss matrix, Bernoulli matrix, part of Hadamard matrix, sparse random matrix, QC-LDPC matrix. The different measurement matrix simulation results are shown in Figure 8.The PSNR (Peak Signal to Noise Ratio), MSE(Mean Square Error) and MD (Matching Degree) of the reconstruction measurement matrix are shown in Table 2.

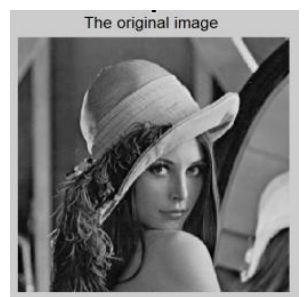

(a) The original image

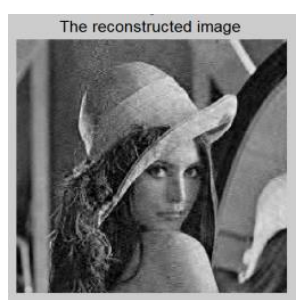

(d) part of Hadamard matrix

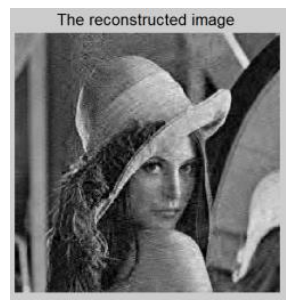

(b) Gauss matrix

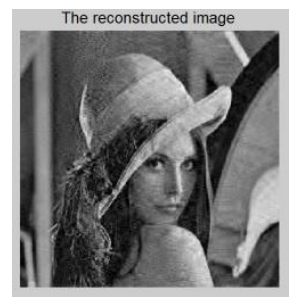

(e) sparse random matrix

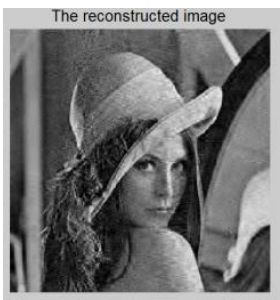

(c) Bernoulli matrix

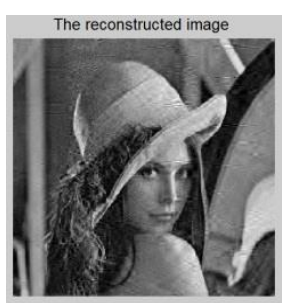

(f) QC-LDPC matrix

Figure 8. Two-Dimensional Signal Reconstruction by Measurement Matrix

Table 2. PSNR, MSE and MD of the Reconstruction Measurement Matrix

\begin{tabular}{cccc}
\hline Measurement matrix & PSNR(dB) & MSE & MD \\
\hline Gauss matrix & 26.6885 & 1.393 & $97.75 \%$ \\
\hline Bernoulli matrix & 26.5405 & 1.442 & $96.38 \%$ \\
\hline part of Hadamard matrix & 27.3656 & 1.192 & $98.52 \%$ \\
\hline Sparse random matrix & 26.5645 & 1.434 & $96.44 \%$ \\
\hline QC-LDPC matrix & 26.8402 & 1.346 & $98.13 \%$ \\
\hline
\end{tabular}

In Table 2, the simulation results can be seen that the proposed QC-LDPC matrix is better than the Gaussian random matrix as the measurement matrix. Moreover, the structure of Gaussian random matrix and other random matrix are uncertain, which lead to its implementation difficulty, and require huge storage resources. The matrix structure of 
QC-LDPC is deterministic structure, which reduce the complexity of the matrix structure and storage resources. In addition, the proposed measurement matrix has quasi cyclic properties and sparse characteristics in the structure, which greatly reduce the storage space requirements and the complexity of the hardware implementation.

\section{Conclusion}

In practical applications, the measurement matrix is not only has high reconstruction accuracy, but also should have less number, low computation complexity, less storage space, easy to hardware implementation, etc. In this paper, a new structured sparse matrix measurement is proposed based on QC-LDPC matrix. The measurement matrix is very sparse, symmetry and circulation. It can be not only save the storage space for storage matrix element, but also can be obtained the better reconstruction performance of Gauss random measurement matrix. The next job is to use FPGA devices to achieve the measurement matrix in the further.

\section{Acknowledgement}

We would like to thank my mentor Professor Yang for stimulating discussions with respect to the topic of this paper and laboratory equipment. Moreover, we greatly appreciate the reviewers' comments that lead to an improved presentation of the results. This work was supported by Research Program of science and technology at Universities of Inner Mongolia Autonomous Region (NJZZ14288).

\section{References}

[1] E. J. Candes and T. Tao, "Decoding by linear programming," IEEE Trans. Inf. Theory, vol. 51, no. 12, (2005), pp. 4203-4215.

[2] D. Donoho, “Compressed sensing," IEEE Trans. Inf. Theory, vol. 52, no. 4, (2006), pp. 1289-1306.

[3] E. J. Candes and M. B. Wakin, "An introduction to compressive sampling," ' IEEE Signal Proc. Mag., vol. 25, no. 2, (2008), pp. 21-30.

[4] R. A. DeVore, "Deterministic constructions of compressed sensing matrices," J. Complexity, vol. 23, no. 4-6, (2007), pp. 918-925.

[5] E. J. Candes, "The restricted isometry property and its implications for compressed sensing," ComptesRendusMathematique, vol .346, no.8-9, (2008), pp. 589-592.

[6] R. G. Gallager, "Low density parity check codes", IRE Trans. Inf. Theory, vol. IT-8, (1962), pp. 21-28.

[7] L. Chen, J. Xu, I. Djurdjevic, and S. Lin, "Near-Shannon-limit quasi-cyclic low-density parity-check codes", IEEE Trans. Commun, vol. 52, no. 7,(2003), pp. 2030-2035.

[8] H. Xiao, and A. H. Banihashemi, "Improved progressive-edge-growth (PEG) construction of irregular LDPC codes," IEEE Communications Letters, vol. 8, no. 12, (2004), pp. 715-717.

[9] R. A. DeVore, "Deterministic constructions of compressed sensing matrices," J. Complexity, vol. 23, no. 4-6, (2007), pp. 918-925.

[10] A. Gilbert and P. Indyk, "Sparse recovery using sparse matrices," Proceedings of the IEEE, vol. 98, no. 6, (2010), pp. 937-947.

[11] R. Baraniuk, M. Davenport, R. DeVore, and M. Wakin, "A simple proof of the restricted isometry property for random matrices," Constructive Approximation, vol. 28, no. 3, (2008), pp. 253-263.

[12] J. A. Tropp and A. C. Gilbert, "Signal recovery from random measurements via orthogonal matching pursuit," IEEE Trans. Inf. Theory,vol. 53, no. 12, (2007), pp. 4655-4666.

[13] E. Candes, J. Romberg, and T. Tao, "Stable Signal Recovery from Incomplete and Inaccurate".

[14] Measurements", Communications on Pure and Applied Mathematics, vol. 59, no. 8, (2006), pp. 12071223.

[15] J. Haupt, R. Nowak, "Signal reconstruction from noisy random projections," IEEE Trans. Info.Theory, vol. 52, no. 9, (2006), pp.4036-4048.

[16] M. Rudelson and R. Vershynin, "On sparse reconstruction from Fourier and Gaussian measurements," Comm. Pure Appl. Math, vol. 61, no. 8, (2008), pp. 1025-1171.

[17] Y. Rivenson and A. Stern, "Compressed imaging with a separable sensing operator," IEEE Signal Proc. Letters, vol. 16, no. 6, ( 2009), pp.449-452.

[18] S. Gazit, A. Szameit, Y. C. Eldar, and M. Segev, "Super-resolution and reconstruction of sparse subwavelength images,” Opt.Express, vol. 17, (2009), pp. 23920-23946. 
[19] L. He and L. Carin, "Exploiting structure in wavelet-based Bayesian compressive sensing," IEEE Trans. Signal Proc., vol. 57, no.9, (2009), pp. 3488-3497.

\section{Authors}

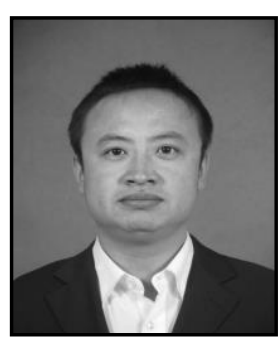

NIE Yang, He is a Ph.D. in the Digital Engineering Center of Communication University of China and is a lecturer of Jining Normal University, China. His main research interests include Compressive Sensing, High-performance DSP Algorithms and VLSI Architectures.

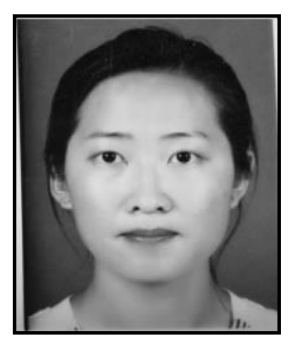

JING Li-li, She is a lecturer of Jining Normal University, China. Here main research interests include Compressive Sensing, Highperformance DSP Algorithms. 